\title{
Multiple Markers of Contrast Induced Nephropathy after the Percutaneous Coronary Intervention
}

\author{
Byoung-won Park', Seong Soon Kwon' ${ }^{1}$, Min Ho Lee', Do Hoi Kim², Min Su Hyon', Duk Won Bang ${ }^{1}$ \\ 'Division of Cardiology, Department of Internal Medicine, Soonchunhyang University Hospital, Seoul; ${ }^{2}$ Department of Internal Medicine, Uvis Hospital, \\ Incheon, Korea
}

\begin{abstract}
Objective: Contrast-induced nephropathy (CIN) frequently occurs after percutaneous intervention. Objective of this study was to investigate the usefulness of serum cystatin C, neutrophil gelatinase-associated lipocalcin (NGAL), urinary kidney injury molecule-1 (KIM-1), and interleukin-18 (IL-18) as early predictors for CIN after percutaneous coronary intervention (PCI).

Methods: In 53 patients who underwent PCI were enrolled. Serum creatinine and cystatin C level were measured immediately before, and 24 hours and 48 hours after catheterization. Serum NGAL, urinary KIM-1, and IL-18 were measured immediately before, and 4 hours, 24 hours, and 48 hours after catheterization. CIN was defined as a rise in creatinine $0.5 \mathrm{mg} / \mathrm{dL}$ or $25 \%$ above baseline. Results: CIN occurred in four patients (7.5\%). Serum cystatin C levels were higher at 24 hours and 48 hours in CIN patients than in those without CIN $(P<0.05)$. Serum NGAL levels were higher at 48 hours in CIN patients than in those without CIN. Urinary KIM-1 levels were higher at 48 hours in CIN patients than in those without CIN. There were no significant markers of CIN on multi-variate analysis.

Conclusion: In this study, the occurrence of $\mathrm{CIN}$ after PCI was 7.5\%. Although there were some time-course changes in serum cystatin $\mathrm{C}$ and urinary KIM-1 after PCl, there was no significant predictor for CIN after PCl.
\end{abstract}

Keywords: Nephropathy; Percutaneous coronary intervention; Contrast media

\section{INTRODUCTION}

Contrast-induced nephropathy (CIN) is responsible for more than $10 \%$ of acute renal failure developed in hospitalized patients after diagnostic tests using contrast media. The manifestation of CIN is directly increased as the number of diagnostic tests or procedures using contrast media rises [1-3].

$\mathrm{CIN}$ is one of the causes of prolonged hospitalization, increased morbidity, and worsening renal insufficiency leading to chronic renal failure which sometimes requires dialysis [4]. Although the incidence of CIN is less than $1 \%$ in normal population, it has been reported in $5.5 \%$ of patients who have preexisting renal insufficiency and it is increased up to $50 \%$ of patient if they have diabetes $[1,2]$.

Several laboratory markers to predict CIN after coronary angi- ography have been reported. Cystatin C (CysC) [5-7], neutrophil gelatinase-associated lipocalcin (NGAL) [8-10], urinary kidney injury molecule-1 (KIM-1) [11,12], and interleukin-18 (IL-18) [1215] have been studied and have showed reliable results.

Also, predictive risk factors for CIN have been well recognized. In a study reporting a risk score for prediction of $\mathrm{CIN}$ after percutaneous coronary intervention (PCI), several clinical factors such as hypotension, congestive heart failure, age, anemia, diabetes, amount of contrast media used, estimated glomerular filtration rate (GFR), and use of intra-aortic balloon pump have been reported [16].

The purpose of this study was to identify the prevalence and clinical features of CIN among patients underwent $\mathrm{PCI}$ and evaluate the usefulness of the serum $\mathrm{CysC}$, serum NGAL, urinary KIM-1, and urinary IL-18 as the early predictors for CIN by mea- 
Park BW, et al. • Multiple Markers of Contrast Induced Nephropathy

suring of each marker serially.

\section{MATERIALS AND METHODS}

A total of 53 patients who underwent PCI for stable angina at Soonchunhyang University Seoul Hospital were enrolled from May 2009 to November 2009. This study was approved by the Institutional Review Board of Soonchunhyang University Seoul Hospital (IRB approval no., 2009-042). Each participant provided written informed consent. Exclusion criteria were as follows: (1) serum creatinine level (Cr) more than $2.0 \mathrm{mg} / \mathrm{dL}$; (2) GFR less than $30 \mathrm{~mL} / \mathrm{min} / 1.73 \mathrm{~m}^{2}$ at stable condition; (3) chronic renal failure patients who is on hemodialysis; (4) multiple myeloma; (5) pulmonary edema; (6) uncontrolled hypertension (systolic blood pressure [BP] more than $160 \mathrm{~mm} \mathrm{Hg}$ or diastolic BP more than $100 \mathrm{~mm} \mathrm{Hg}$ ); (7) a history using contrast media within 2 days; (8) allergic reaction to contrast media; (9) pregnancy; and (10) patients who were taking theophylline, dopamine, mannitol, fenoldopam, and $\mathrm{N}$-acetylcysteine.

CIN was defined as increase of serum Cr $0.5 \mathrm{mg} / \mathrm{dL}$ or $25 \%$ above baseline within 48 hours after using contrast media [17]. GFR was calculated by modification of diet in renal disease equation [18]. Hypertension was defined as systolic BP more than 140 $\mathrm{mm} \mathrm{Hg}$ and/or diastolic BP more than $90 \mathrm{~mm} \mathrm{Hg}$ at resting and/ or treatment with antihypertensive medication. Diabetes was also defined as fasting blood glucose more than $126 \mathrm{mg} / \mathrm{dL}$ and/or treatment with oral hypoglycemic agents. Left ventricular systolic function was determined through transthoracic echocardiography by measurement of left ventricular ejection fraction.

Coronary angiography was performed using standard techniques and interventional instruments were decided by operator's discretion. Non-ionic low osmolar contrast media was used regardless of the company of product. Intravenous $0.9 \%$ isotonic saline, as an infusion rate of $1 \mathrm{~mL} / \mathrm{kg} / \mathrm{hr}$, was given before, and after 12 hours of procedure for all patients.

We performed laboratory test including lipid profile, pro-brain natriuretic peptide, and C-reactive protein before PCI. Serum $\mathrm{Cr}$ was measured immediately before and, 4, 24, and 48 hours after PCI. The serum CysC was measured by ELISA kit (Dade Behring, Deerfield, IL, USA) and serum NGAL by ELISA kit (BioPorto, Gentofte, Denmark) immediately before, 4, 24, and 48 hours after $\mathrm{PCI}$, which were stored in $-70^{\circ} \mathrm{C}$ refrigerator after centrifugation. Fresh urine samples collected early in the morning and stored at $-70^{\circ} \mathrm{C}$ refrigerator were centrifuged to remove cellular components. Urinary KIM-1 and IL-18 were measured just before, 4, 24, and 48 hours after PCI and analyzed by ELISA kit (R\&D Systems, Minneapolis, MN, USA). Urinary KIM-1 and IL-18 were adjusted with urinary $\mathrm{Cr}$ in the early morning spot urine.

Data are presented as numbers and frequencies for categorical variables and as mean \pm standard deviation for continuous variables. For comparisons between the patients with and without CIN, independent sample t-test for continuous variables and chisquare test was used for categorical variables. The changes of each marker were analyzed by repeated measures analysis of variance with Bonferroni test. Correlations between continuous variables were analyzed by the Pearson's correlation. To identify the independent predictive factors for $\mathrm{CIN}$, univariate and multivariate logistic regression was used. All statistical analyses were conducted with the SPSS ver. 13.0 (SPSS, Chicago, IL, USA) and two-sided Pvalues $<0.05$ were considered statistically significant.

Table 1. Baseline characteristics of patients

\begin{tabular}{|c|c|c|c|}
\hline Characteristic & $\mathrm{CIN}(+)(\mathrm{n}=4)$ & CIN $(-)(n=49)$ & P-value \\
\hline Age (yr) & $64 \pm 14$ & $65 \pm 11$ & 0.80 \\
\hline Sex (male/female) & $2 / 2$ & $29 / 20$ & 0.70 \\
\hline Body mass index (kg/m²) & $30 \pm 3$ & $24 \pm 3$ & 0.001 \\
\hline Hypertension & $3(75)$ & $24(48)$ & 0.30 \\
\hline Diabetes & $2(50)$ & $13(26)$ & 0.30 \\
\hline Contrast volume (mL) & $198 \pm 53$ & $202 \pm 62$ & 0.92 \\
\hline Hematocrit & $36 \pm 4$ & $37 \pm 5$ & 0.78 \\
\hline Total cholesterol (mg/dL) & $232 \pm 82$ & $164 \pm 34$ & 0.001 \\
\hline Triglyceride (mg/dL) & $352 \pm 383$ & $146 \pm 65$ & 0.001 \\
\hline $\begin{array}{l}\text { High density lipoprotein cholesterol } \\
\text { (mg/dL) }\end{array}$ & $37 \pm 3$ & $41 \pm 7$ & 0.31 \\
\hline $\begin{array}{l}\text { Low-density lipoprotein cholesterol } \\
\text { (mg/dL) }\end{array}$ & $131 \pm 41$ & $98 \pm 26$ & 0.03 \\
\hline Hemoglobin A1c (\%) & $7.7 \pm 2.6$ & $6.6 \pm 1.2$ & 0.13 \\
\hline $\begin{array}{l}\text { Angiotensin converting enzyme } \\
\text { inhibitor }\end{array}$ & $1(25)$ & $7(14)$ & 0.55 \\
\hline Angiotensin receptor blocker & 0 & $11(22)$ & 0.29 \\
\hline Beta blocker & $3(75)$ & $15(30)$ & 0.07 \\
\hline Statin & $3(75)$ & $46(92)$ & 0.26 \\
\hline Ca-channel blocker & $2(50)$ & $18(36)$ & 0.58 \\
\hline Brain natriureptic peptide (ng/mL) & $1,011 \pm 803$ & $529 \pm 1,156$ & 0.49 \\
\hline Ejection fraction (\%) & $57 \pm 10$ & $59 \pm 22$ & 0.82 \\
\hline $\begin{array}{l}\text { Estimated glomerular filtration rate } \\
(\mathrm{mL} / \mathrm{min})\end{array}$ & $69.1 \pm 29.7$ & $86.2 \pm 27.1$ & 0.23 \\
\hline
\end{tabular}

Values are presented as mean \pm standard deviation or number $(\%)$. CIN, contrast induced nephropathy. 


\section{RESULTS}

\section{Patient characteristics}

A total of 53 patients, CIN occurred in four patients (7.5\%). Baseline characteristics were shown at Table 1. There were no significant differences in age, sex, hypertension, and diabetes between patients with CIN (CIN group) and without CIN (non-CIN group). Also, contrast volume in CIN group was not different from nonCIN groups (198 mL vs. $202 \mathrm{~mL}, \mathrm{P}=0.92$ ). However, body mass index (BMI) in CIN group was significantly higher than non-CIN group $\left(30 \pm 3 \mathrm{~kg} / \mathrm{m}^{2}\right.$ vs. $\left.24 \pm 3 \mathrm{~kg} / \mathrm{m}^{2}, \mathrm{P}=0.001\right)$.

There were no significant differences between the groups on laboratory findings and drug history except lipid profile. Total cholesterol, triglyceride, and low-density lipoprotein (LDL) cholesterol in CIN group were significantly higher than non-CIN group $(232 \pm 82 \mathrm{mg} / \mathrm{dL}$ vs. $164 \pm 34 \mathrm{mg} / \mathrm{dL}, \mathrm{P}=0.001 ; 352 \pm 383$ $\mathrm{mg} / \mathrm{dL}$ vs. $146 \pm 65 \mathrm{mg} / \mathrm{dL}, \mathrm{P}=0.001 ; 131 \pm 41 \mathrm{mg} / \mathrm{dL}$ vs. $98 \pm 26$ $\mathrm{mg} / \mathrm{dL}, \mathrm{P}=0.03$, respectively)

\section{Glomerular filtration rate, serum creatinine, and cystatin C level}

The baseline GFR and serum Cr showed no difference between two groups. However, the serum $\mathrm{Cr}$, measured at 24 hours after PCI, was significantly increased in CIN group than non-CIN group $(1.13 \pm 0.34 \mathrm{mg} / \mathrm{dL}$ vs. $0.85 \pm 0.24 \mathrm{mg} / \mathrm{dL}, \mathrm{P}<0.05)$. The serum $\mathrm{Cr}$, measured after at 48 hours after $\mathrm{PCI}$, was not different between two groups.

In case of serum CysC level, there was no significant difference at baseline between two groups. However, the serum CysC, measured at 24 and 48 hours after PCI, were significantly higher in CIN group than non-CIN group $(1.58 \pm 0.79 \mathrm{mg} / \mathrm{dL}$ vs. $1.13 \pm$ $0.34 \mathrm{mg} / \mathrm{dL}, \mathrm{P}=0.04 ; 1.62 \pm 0.85 \mathrm{mg} / \mathrm{dL}$ vs. $1.10 \pm 0.36 \mathrm{mg} / \mathrm{dL}$, $\mathrm{P}=0.02$, respectively) (Table 2 ).

\section{The serum neutrophil gelatinase-associated lipocalcin, urinary kidney injury molecule-1, and urinary interleukin-18}

The serial alteration of the serum NGAL, urinary KIM-1, and urinary IL-18 were shown in Table 3. The baseline serum NGAL level in CIN group was not different from non-CIN group. However, serum NGAL in non-CIN group was significantly increased at 24 hours after PCI, as compared with baseline (161.3 $\pm 113.3 \mathrm{ng} /$ $\mathrm{mL}$ vs. $105.0 \pm 73.3 \mathrm{ng} / \mathrm{mL}, \mathrm{P}<0.05)$. However, in CIN group, there was no significant difference (Fig. 1).

The urinary KIM-1 at 24 hours after PCI did not show difference between two groups. But, urinary KIM-1 at 48 hours after PCI was significantly higher in the CIN group than non-CIN group $(1.4 \pm 0.6 \mathrm{ng} / \mathrm{mL}$ vs. $0.7 \pm 0.5 \mathrm{ng} / \mathrm{mL}, \mathrm{P}<0.05)$. The urinary KIM-1 in at 4 hours after PCI in non-CIN group was significantly decreased, compared with at baseline $(0.3 \pm 0.3 \mathrm{ng} / \mathrm{mL}$ vs. $0.5 \pm$ $0.4 \mathrm{ng} / \mathrm{mL}, \mathrm{P}<0.05)$. The urinary KIM-1 were increased at 24 and 48 hours after PCI $(0.8 \pm 0.5 \mathrm{ng} / \mathrm{mL}$ vs. $0.3 \pm 0.3 \mathrm{ng} / \mathrm{mL}, 0.7 \pm 0.5$

Table 2. Time-course changes in serum creatinine, cystatin C

\begin{tabular}{|c|c|c|c|c|c|c|}
\hline \multirow{2}{*}{$\begin{array}{c}\text { Percutaneous coro- } \\
\text { nary intervention }\end{array}$} & \multicolumn{3}{|c|}{ Creatinine (mg/dL) } & \multicolumn{3}{|c|}{ Cystatin C (mg/dL) } \\
\hline & $\mathrm{CIN}(+)$ & CIN (-) & P-value & $\mathrm{CIN}(+)$ & $\operatorname{CIN}(-)$ & P-value \\
\hline Before & $1.0 \pm 0.23$ & $0.87 \pm 0.25$ & 0.30 & $1.45 \pm 0.62$ & $1.10 \pm 0.39$ & 0.11 \\
\hline After $24 \mathrm{hr}$ & $1.13 \pm 0.28$ & $0.85 \pm 0.24$ & 0.03 & $1.58 \pm 0.79$ & $1.13 \pm 0.34$ & 0.04 \\
\hline After $48 \mathrm{hr}$ & $1.18 \pm 1.40$ & $0.89 \pm 0.30$ & 0.08 & $1.62 \pm 0.85$ & $1.10 \pm 0.36$ & 0.02 \\
\hline
\end{tabular}

Values are presented as mean \pm standard deviation.

$\mathrm{CIN}$, contrast induced nephropathy.

Table 3. Time-course changes in serum NGAL and urinary KIM-1, IL-18

\begin{tabular}{|c|c|c|c|c|c|c|c|c|c|}
\hline \multirow{2}{*}{$\begin{array}{l}\text { Percutaneous coronary } \\
\text { intervention }\end{array}$} & \multicolumn{3}{|c|}{ Serum NGAL (ng/mL) } & \multicolumn{3}{|c|}{ Urinary KIM-1 (ng/mL) } & \multicolumn{3}{|c|}{ Urinary IL-18 (ng/mL) } \\
\hline & $\mathrm{CIN}(+)$ & $\operatorname{CIN}(-)$ & P-value & $\mathrm{CIN}(+)$ & $\operatorname{CIN}(-)$ & P-value & $\mathrm{CIN}(+)$ & $\operatorname{CIN}(-)$ & P-value \\
\hline Before & $92.4 \pm 38.6$ & $105.0 \pm 73.3$ & 0.71 & $0.72 \pm 0.24$ & $0.50 \pm 0.41$ & 0.49 & $2.93 \pm 1.25$ & $3.5 \pm 1.1$ & 0.35 \\
\hline After $4 \mathrm{hr}$ & $107.0 \pm 47.7$ & $153.8 \pm 116.7$ & 0.47 & $0.60 \pm 0.51$ & $0.30 \pm 0.25$ & 0.32 & $3.10 \pm 0.75$ & $3.08 \pm 0.81$ & 0.94 \\
\hline After $24 \mathrm{hr}$ & $117.6 \pm 56.0$ & $161.3 \pm 113.3$ & 0.54 & $1.22 \pm 0.71$ & $0.75 \pm 0.52$ & 0.29 & $3.13 \pm 1.1$ & $3.75 \pm 0.93$ & 0.31 \\
\hline After $48 \mathrm{hr}$ & $216.3 \pm 145.1$ & $143.8 \pm 91.3$ & 0.12 & $1.39 \pm 0.60$ & $0.72 \pm 0.52$ & 0.01 & $3.17 \pm 1.94$ & $3.79 \pm 1.21$ & 0.39 \\
\hline
\end{tabular}

Values are presented as mean \pm standard deviation.

NGAL, neutrophil-gelatinase-associated lipocalcin; KIM-1, kidney injury molecule-1; IL-18, interleukin-18; CIN, contrast induced nephropathy. 


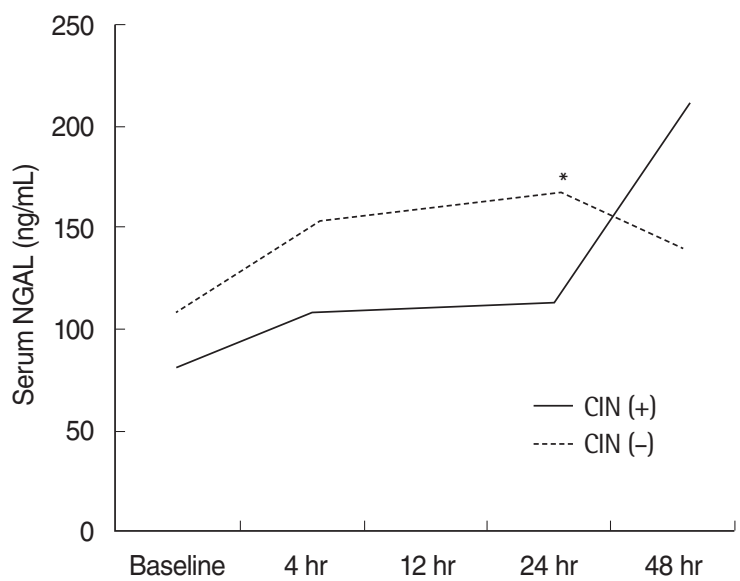

Fig. 1. Time-course changes in serum NGAL. NGAL, neutrophil-gelatinase-associated lipocalcin; $\mathrm{CIN}$, contrast induced nephropathy. ${ }^{*} \mathrm{P}<0.05$ (vs. baseline).

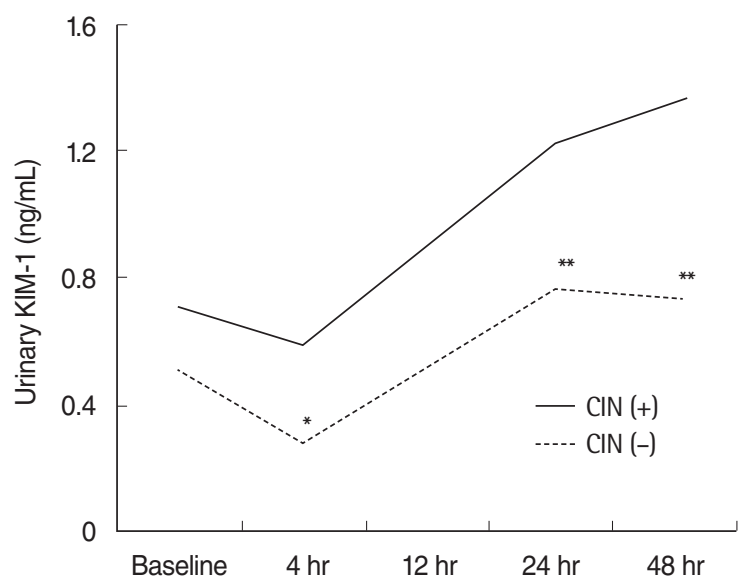

Fig. 2. Time-course changes in urinary KIM-1. KIM-1, kidney injury molecule-1; CIN, contrast induced nephropathy. ${ }^{*} \mathrm{P}<0.05$ (vs. baseline). ${ }^{*} \mathrm{P}<0.05$ (vs. after $4 \mathrm{hr})$.

$\mathrm{ng} / \mathrm{mL}$ vs. $0.3 \pm 0.3 \mathrm{ng} / \mathrm{mL}$, respectively; $\mathrm{P}<0.05)$. In contrast, time-course changes were not found in CIN group (Fig. 2).

The urinary IL-18 at 24 and 48 hours after PCI was significantly higher in the non-CIN group compared with at 4 hours (Fig. 3). However, time-course changes were not found in CIN group.

\section{Predictive factors for contrast-induced nephropathy by univariate and multivariate logistic regression analysis}

In univariate logistic regression analysis, $\mathrm{BMI}$ (odds ratio [OR], 1.7; $\mathrm{P}=0.013)$, total cholesterol ( $\mathrm{OR}, 1.03 ; \mathrm{P}=0.035)$, 48 hours CysC (OR, 8.22; $\mathrm{P}=0.041)$, and 4 hours urinary KIM-1 (OR, 9.4; $\mathrm{P}=0.04$ ) were significant variables for CIN (Table 4). However, there was no significant predictor for CIN in multivariate analysis.

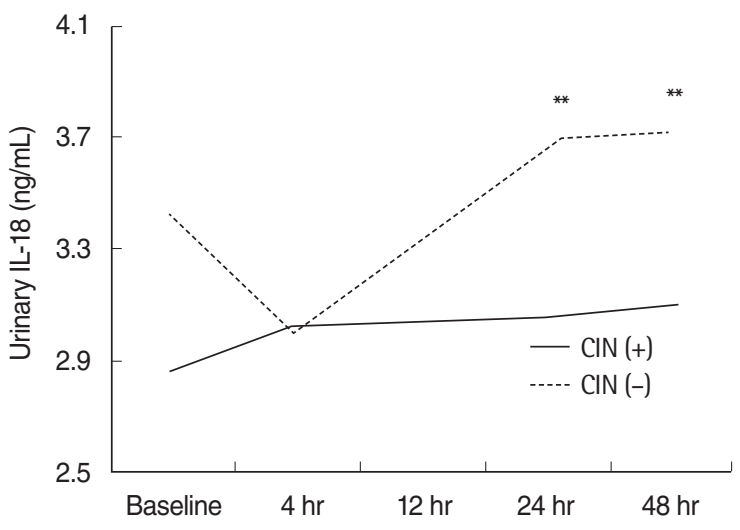

Fig. 3. Time-course changes in urinary IL-18. IL-18, interleukin-18; CIN, contrast induced nephropathy. ${ }^{*} \mathrm{P}<0.05$ (vs. after $4 \mathrm{hr}$ ).

Table 4. Univariate logistic regression analysis of variables related to contrast induced nephropathy in all patients

\begin{tabular}{lcc}
\hline & \multicolumn{2}{c}{ Univariate analysis } \\
\cline { 2 - 3 } Variable & $\begin{array}{c}\text { Odds ratio } \\
\text { (95\% confidence interval) }\end{array}$ & P-value \\
\hline Age & $0.99(0.9-1.01)$ & 0.79 \\
Hypertension & $3.4(0.33-34.9)$ & 0.31 \\
Diabetes & $3.1(0.39-24.3)$ & 0.29 \\
Body mass index (kg/m²) & $1.7(1.13-2.7)$ & 0.01 \\
Total cholesterol & $1.03(1.00-1.06)$ & 0.04 \\
Triglyceride & $1.01(0.99-1.02)$ & 0.13 \\
Low-density lipoprotein cholesterol & $1.04(1.00-1.08)$ & 0.05 \\
Beta blocker & $7.5(0.72-78.4)$ & 0.09 \\
Statin & $0.27(0.02-3.2)$ & 0.30 \\
Estimated glomerular filtration rate (mL/min) & $0.97(0.93-1.02)$ & 0.24 \\
Creatinine (mg/mL) & & \\
Baseline & $6.2(0.19-206.5)$ & 0.31 \\
$24 \mathrm{hr}$ & $48.5(0.95-2476.8)$ & 0.05 \\
$48 \mathrm{hr}$ & $10.6(0.6-184.9)$ & 0.11 \\
Cystatin C (mg/mL) & & \\
Baseline & & \\
$24 \mathrm{hr}$ & $6.5(0.62-68.1)$ & 0.12 \\
$48 \mathrm{hr}$ & $8.43(0.83-85.5)$ & 0.07 \\
Neutrophil-gelatinase-associated lipocalcin & $0.22(1.09-61.9)$ & 0.04 \\
(ng/mL) & $0.99(0.98-1.02)$ & 0.71 \\
Kidney injury molecule-1 (ng/mL) & & \\
Baseline & & \\
$24 \mathrm{hr}$ & $2.0(0.27-15.5)$ & 0.50 \\
Interleukin-18 (ng/mL) & $9.4(1.1-80.87)$ & 0.04 \\
& $0.7(0.34-1.5)$ & 0.35 \\
\hline
\end{tabular}

\section{DISCUSSION}

The major findings of the present analyses are as follows. (1) Among 53 patients who underwent PCI, four patients (7.5\%) were 
developed the CIN. (2) Baseline BMI, total cholesterol, LDL-cholesterol, and triglyceride in CIN-group were higher than non-CIN group. (3) Serum CysC levels at 24 hours and 48 hours after PCI were higher in CIN group. (4) Serum NGAL was increased in 4 hours after PCI and reached to peak level at 24 hours PCI and begun to decrease at 48 hours after PCI. (5) Urinary KIM-1 and urinary IL-18 were temporally decreased 4 hours after PCI and then reached to peak level at 24 hours and begun to decrease at 48 hours after PCI, especially in CIN-group.

\section{Prevalence and clinical features of contrast-induced nephropathy}

The intravascular injection of contrast media is a common cause of CIN. It is well known that CIN is associated with prolonged hospitalization, increased mortality, and prolonged renal injury after coronary angiography or repeated PCI [4]. The development of CIN is low in patients with normal renal function, varying from $0 \%$ to $10 \%$ [19]. However, its incidence is increased to $20 \%$ in case of patients whose baseline serum $\mathrm{Cr}$ was between 1.5 and $2.0 \mathrm{mg} / \mathrm{dL}[12]$.

\section{Usefulness of cystatin $C$ and neutrophil gelatinase- associated lipocalcin in contrast-induced nephropathy}

In chronic kidney disease patients, it needs to try to prevent development of CIN. But, it is difficult to assess the risk of CIN only by serum Cr. Although serum $\mathrm{Cr}$ is a standard test for an acute renal injury, it is not accurately correlated with acute renal dysfunction. Especially, in acute renal injury, serum Cr was not elevated for several days after beginning of renal tubular damage [13]. Though Coca et al. [20] reported about 21 serum and urine makers for diagnosis of acute renal injury by systematically review of 31 studies related with acute renal injury, more investigations are needed to evaluate their effectiveness. Fortunately, many studies are conducted regarding to serum CysC, urinary NGAL, IL-18, KIM-1, and L-type fatty acid binding protein, recently [14,15].

Serum CysC is one of the promising surrogate markers for GFR and it could be superior to serum Cr. It is useful in old ages because it is well correlated with renal dysfunction regardless of age and not affected by sex, and muscle mass [16,17]. Kimmel et al. [21] also reported that $\mathrm{CysC}$ is a more reliable marker than serum $\mathrm{Cr}$ for prediction of CIN in 54 patients who underwent coronary angiography.

NGAL is known to be a predicted marker for acute ischemic re- nal injury. Mishra et al. [22] studied 20 patients who developed acute renal injury among 71 patients who received cardiopulmonary bypass for congenital heart disease surgery. According to the study, acute renal injury was affected by serum and urinary NGAL at 2 hours after surgery, and operation time and urinary NGAL was most reliable independent maker for acute renal injury in univariate analysis. However, in our study, there was no relation between NGAL and acute renal injury. It might be explained by limited numbers of CIN patients in our study. Although CIN is a serious complication after coronary angiography and PCI, it is difficult to assess acute renal injury only with serum $\mathrm{Cr}$ or $\mathrm{Cr}$ clearance because patients are routinely discharged in 48 hours.

In a study, conducted to angina pectoris patients who had normal Cr after coronary angiography and PCI, serum and urinary CysC and NGAL were checked before, 2, 4, 8, 24, and 48 hours after intervention [23]. Among them, CIN was occurred in 11\%, and serum and urinary NGAL were highest at 2, 4 hours after PCI. In contrast, CysC in CIN patients was slightly higher at 8, 24 hours after PCI.

It has previously reported that NGAL is highly sensitive and it can function as an early prognostic marker for acute renal injury after PCI. Elevated NGAL caused by inflammatory reaction of neutrophil after PCI. NGAL is increased after PCI because it is increased in atheromatous plaque [24]. NGAL showed the maximum elevation after 24 hours in non-CIN group. However CysC and serum Cr were not elevated in this study.

\section{Usefulness of kidney injury molecule-1 in cystatin C}

KIM-1 is a transmembrane tubular protein which increases dramatically after injury in proximal tubule epithelial cells during ischemic and toxic renal injury [25]. It has been used as a new marker for diagnosis of acute renal injury [26]. It has been reported that urinary KIM-1 might serve as a useful biomarker for renal proximal tubular injury facilitating the early diagnosis of the disease because it was significantly increased in patients with acute ischemic tubular necrosis compared with chronic renal failure or other acute renal injury [11]. In this study, KIM-1 was significantly increased at 24 and 48 hours after contrast injection in non-CIN group and 48 hours KIM-1 was higher in CIN group compared with non-CIN group. Yet, it needs larger studies in order to confirm KIM-1 as a prognostic marker for renal injury because it only showed significant difference in non-CIN group. 
Park BW, et al. • Multiple Markers of Contrast Induced Nephropathy

\section{Usefulness of interleukin-18 in cystatin C}

In a study conducted with $13 \mathrm{CIN}$ group and 27 non-CIN group after coronary angiography, urinary IL-18 was significantly increased in the CIN group and independent predictive marker for later major cardiac events [13]. However, another study with 15 CIN and 36 non-CIN group after PCI, urinary IL-18 was not significantly different [14]. In our study, IL-18 was significantly increased at 24 and 48 hours after PCI compared with 4 hours after PCI. But it needs more studies to confirm that IL-18 is affected by renal injury actually, because IL-18 was increased 24 and 48 hours after PCI even in non-CIN group. Since most studies regarding IL18 with renal injury were relatively small-sized studies, it needs larger studies to identify urinary IL-18 as an early predictor for CIN.

\section{Limitations}

The limitations of this study are as follows. First, the number of patients, especially CIN patients was relatively small. Second, though patients with normal renal function were studied, variable distribution of GFR by multiple factors was related with analysis of result. Later on, it needs to identify in terms of influence of renal dysfunction with large numbers of patient who is divided group by GFR. Third, it was often difficult to verify consistency about results from process of samples which were affected by many factors, such as obtaining samples, centrifuge, and cold storage.

\section{Conclusion}

In this study, the occurrence of CIN after PCI was 7.5\%. Although there were some time-course changes in serum $\mathrm{CysC}$ and urinary KIM-1 after PCI, there was no significant predictor for CIN after PCI.

\section{REFERENCES}

1. Artunc FH, Fischer IU, Risler T, Erley CM. Improved estimation of GFR by serum cystatin $\mathrm{C}$ in patients undergoing cardiac catheterization. Int $\mathrm{J}$ Cardiol 2005; 102:173-8.

2. Schrader R. Contrast material-induced renal failure: an overview. J Interv Cardiol 2005;18:417-23.

3. Koyner JL, Bennett MR, Worcester EM, Ma Q, Raman J, Jeevanandam V, et al. Urinary cystatin $\mathrm{C}$ as an early biomarker of acute kidney injury following adult cardiothoracic surgery. Kidney Int 2008;74:1059-69.

4. Jo SH. N-acetylcysteine for prevention of contrast-induced nephropathy: a narrative review. Korean Circ J 2011;41:695-702.

5. Gambaro G, Graziani MS, Ribichini F. Cystatin C and contrast-induced nephropathy. Nephrol Dial Transplant 2008;23:4079.
6. Ebru AE, Kilic A, Korkmaz FS, Seker R, Sasmaz H, Demirtas S, et al. Is cystatin-C superior to creatinine in the early diagnosis of contrast-induced nephropathy?: a potential new biomarker for an old complication. J Postgrad Med 2014;60:135-40.

7. Briguori C, Visconti G, Rivera NV, Focaccio A, Golia B, Giannone R, et al Cystatin C and contrast-induced acute kidney injury. Circulation 2010; 121:2117-22.

8. Schilcher G, Ribitsch W, Otto R, Portugaller RH, Quehenberger F, Truschnig-Wilders $\mathrm{M}$, et al. Early detection and intervention using neutrophil gelatinase-associated lipocalin (NGAL) may improve renal outcome of acute contrast media induced nephropathy: a randomized controlled trial in patients undergoing intra-arterial angiography (ANTI-CIN Study). BMC Nephrol 2011;12:39.

9. Bachorzewska-Gajewska H, Malyszko J, Sitniewska E, Malyszko JS, Poniatowski B, Pawlak K, et al. NGAL (neutrophil gelatinase-associated lipocalin) and cystatin C: are they good predictors of contrast nephropathy after percutaneous coronary interventions in patients with stable angina and normal serum creatinine? Int J Cardiol 2008;127:290-1.

10. Liebetrau C, Gaede L, Doerr O, Blumenstein J, Rixe J, Teichert O, et al. Neutrophil gelatinase-associated lipocalin (NGAL) for the early detection of contrast-induced nephropathy after percutaneous coronary intervention. Scand J Clin Lab Invest 2014;74:81-8.

11. Han WK, Bailly V, Abichandani R, Thadhani R, Bonventre JV. Kidney injury molecule-1 (KIM-1): a novel biomarker for human renal proximal tubule injury. Kidney Int 2002;62:237-44.

12. Duan SB, Liu GL, Yu ZQ, Pan P. Urinary KIM-1, IL-18 and Cys-c as early predictive biomarkers in gadolinium-based contrast-induced nephropathy in the elderly patients. Clin Nephrol 2013;80:349-54.

13. Ling W, Zhaohui N, Ben H, Leyi G, Jianping L, Huili D, et al. Urinary IL18 and NGAL as early predictive biomarkers in contrast-induced nephropathy after coronary angiography. Nephron Clin Pract 2008;108: c176-81.

14. Bulent Gul CB, Gullulu M, Oral B, Aydinlar A, Oz O, Budak F, et al. Urinary IL-18: a marker of contrast-induced nephropathy following percutaneous coronary intervention? Clin Biochem 2008;41:544-7.

15. Parikh CR, Mishra J, Thiessen-Philbrook H, Dursun B, Ma Q, Kelly C, et al. Urinary IL-18 is an early predictive biomarker of acute kidney injury after cardiac surgery. Kidney Int 2006;70:199-203.

16. Mehran R, Aymong ED, Nikolsky E, Lasic Z, Iakovou I, Fahy M, et al. A simple risk score for prediction of contrast-induced nephropathy after percutaneous coronary intervention: development and initial validation. J Am Coll Cardiol 2004;44:1393-9.

17. Golshahi J, Nasri H, Gharipour M. Contrast-induced nephropathy; a literature review. J Nephropathol 2014;3:51-6.

18. Twomey PJ, Reynolds TM. The MDRD formula and validation. QJM 2006; 99:804-5.

19. Davidson CJ, Hlatky M, Morris KG, Pieper K, Skelton TN, Schwab SJ, et al. Cardiovascular and renal toxicity of a nonionic radiographic contrast agent after cardiac catheterization: a prospective trial. Ann Intern Med 1989;110:119-24.

20. Coca SG, Yalavarthy R, Concato J, Parikh CR. Biomarkers for the diagnosis and risk stratification of acute kidney injury: a systematic review. Kidney Int 2008;73:1008-16.

21. Kimmel M, Butscheid M, Brenner S, Kuhlmann U, Klotz U, Alscher DM. Improved estimation of glomerular filtration rate by serum cystatin $\mathrm{C}$ in preventing contrast induced nephropathy by $\mathrm{N}$-acetylcysteine or zinc: preliminary results. Nephrol Dial Transplant 2008;23:1241-5.

22. Mishra J, Dent C, Tarabishi R, Mitsnefes MM, Ma Q, Kelly C, et al. Neutrophil gelatinase-associated lipocalin (NGAL) as a biomarker for acute 
renal injury after cardiac surgery. Lancet 2005;365:1231-8.

23. Bachorzewska-Gajewska H, Malyszko J, Sitniewska E, Malyszko JS, Dobrzycki S. Neutrophil gelatinase-associated lipocalin (NGAL) correlations with cystatin C, serum creatinine and eGFR in patients with normal serum creatinine undergoing coronary angiography. Nephrol Dial Transplant 2007;22:295-6.

24. Hemdahl AL, Gabrielsen A, Zhu C, Eriksson P, Hedin U, Kastrup J, et al. Expression of neutrophil gelatinase-associated lipocalin in atherosclero- sis and myocardial infarction. Arterioscler Thromb Vasc Biol 2006;26: 136-42.

25. Ichimura T, Hung CC, Yang SA, Stevens JL, Bonventre JV. Kidney injury molecule-1: a tissue and urinary biomarker for nephrotoxicant-induced renal injury. Am J Physiol Renal Physiol 2004;286:F552-63.

26. Van Timmeren MM, van den Heuvel MC, Bailly V, Bakker SJ, van Goor $\mathrm{H}$, Stegeman CA. Tubular kidney injury molecule-1 (KIM-1) in human renal disease. J Pathol 2007;212:209-17. 